\title{
Obesity phenotype is related to NLRP3 inflammasome activity and immunological profile of visceral adipose tissue
}

\author{
Nathalie Esser • Laurent L'homme • Arnaud De Roover • \\ Laurent Kohnen • André J. Scheen • Michel Moutschen • \\ Jacques Piette • Sylvie Legrand-Poels • Nicolas Paquot
}

Received: 5 April 2013 / Accepted: 23 July 2013 / Published online: 7 September 2013

(C) Springer-Verlag Berlin Heidelberg 2013

\begin{abstract}
Aims/hypothesis Obesity is a heterogeneous condition comprising both individuals who remain metabolically healthy (MHO) and those who develop metabolic disorders (metabolically unhealthy, MUO). Adipose tissue is also heterogeneous in that its visceral component is more frequently associated with metabolic dysfunction than its subcutaneous component. The development of metabolic disorders is partly mediated by the NLR family pyrin domain containing-3 (NLRP3) inflammasome, which increases the secretion of inflammatory cytokines via activation of caspase-1. We compared the immunological profile and NLRP3 activity in adipose tissue between MUO and MHO individuals.

Methods MHO and MUO phenotypes were defined, respectively, as the absence and the presence of the metabolic syndrome. Cellular composition and intrinsic inflammasome activity were investigated by flow cytometry, quantitative RTPCR and tissue culture studies in subcutaneous and visceral
\end{abstract}

Sylvie Legrand-Poels and Nicolas Paquot contributed equally to this work.

Electronic supplementary material The online version of this article (doi:10.1007/s00125-013-3023-9) contains peer-reviewed but unedited supplementary material, which is available to authorised users.

N. Esser $\cdot$ L. L'homme $\cdot$ J. Piette $\cdot$ S. Legrand-Poels $\cdot$ N. Paquot Virology and Immunology Unit, GIGA-ST, University of Liege, Liege, Belgium

N. Esser • A. J. Scheen $\cdot$ N. Paquot $(\square)$

Division of Diabetes, Nutrition and Metabolic Disorders, Department of Medicine, CHU Sart Tilman, B-4000 Liege, Belgium

e-mail: Nicolas.Paquot@ulg.ac.be

A. De Roover $\cdot$ L. Kohnen

Department of Abdominal Surgery and Transplantation, University

Hospital of Liege, Liege, Belgium

M. Moutschen

Immunology and Infectious Diseases Unit, GIGA-I ${ }^{3}$,

University of Liege, Liege, Belgium adipose tissue from $23 \mathrm{MUO}, 21 \mathrm{MHO}$ and nine lean individuals.

Results We found significant differences between the three study groups, including an increased secretion of IL- $1 \beta$, increased expression of $I L 1 B$ and $N L R P 3$, increased number of adipose tissue macrophages and decreased number of regulatory $\mathrm{T}$ cells in the visceral adipose tissue of MUO patients compared with $\mathrm{MHO}$ and lean participants. In macrophages derived from visceral adipose tissue, both caspase-1 activity and IL-1 $\beta$ levels were higher in MUO patients than in MHO patients. Furthermore, caspase-1 activity was higher in $\mathrm{CD} 11 \mathrm{c}^{+} \mathrm{CD} 206^{+}$adipose tissue macrophages than in $\mathrm{CD} 11 \mathrm{c}^{-} \mathrm{CD} 206^{+}$cells.

Conclusions/interpretation The MUO phenotype seems to be associated with an increased activation of the NLPR3 inflammasome in macrophages infiltrating visceral adipose tissue, and a less favourable inflammatory profile compared with the MHO phenotype.

Keywords Adipose tissue $\cdot$ Inflammatory cytokines · Macrophages · Metabolic disorders · NLRP3 inflammasome · Obesity

\begin{tabular}{ll}
\multicolumn{2}{l}{ Abbreviations } \\
APC & Allophycocyanin \\
ATM & Adipose tissue macrophage \\
HOMA-IR & HOMA of insulin resistance \\
MHO & Metabolically healthy obese \\
MUO & Metabolically unhealthy obese \\
NLR & NOD-like receptor \\
NLRP3 & NLR family pyrin domain containing-3 \\
PE & Phycoerythrin \\
PEC5 & Phycoerythrin-cyanin 5 \\
PerCP & Perdidin chlorophyll protein \\
SAT & Subcutaneous adipose tissue \\
SVC & Stromovascular cell \\
VAT & Visceral adipose tissue
\end{tabular}




\section{Introduction}

Obesity is a heterogeneous condition with no direct relationship between body weight and metabolic abnormalities. Approximately $30 \%$ of all obese individuals do not have the metabolic syndrome and are at lower risk of developing type 2 diabetes and cardiovascular diseases. These individuals are referred to in the literature as 'metabolically healthy obese' (MHO), whereas obese individuals with metabolic abnormalities are termed 'metabolically unhealthy obese' (MUO) [1-5]. Similarly, white adipose tissue is also heterogeneous, consisting of a peripheral subcutaneous component (subcutaneous adipose tissue [SAT]) and a central intra-abdominal component (visceral adipose tissue [VAT]); these components differ in phenotypic, physiological and functional characteristics $[6,7]$. Although both SAT and VAT are associated with metabolic risk profile, it is reported that high VAT is more strongly correlated with the metabolic syndrome than is SAT $[8,9]$. However, excess VAT may be a marker of dysfunctional SAT not being able to expand when facing an energy surplus [7]. Indeed, in addition to the amount and distribution of fat, subcutaneous adipocyte hypertrophy is an independent marker of insulin resistance [10] and a predictor of type 2 diabetes [11]. Interestingly, hypertrophic adipocytes have a reduced lipid-buffering capacity and a more pro-inflammatory phenotype [12].

Chronic low-grade inflammation is observed among individuals with visceral obesity and represents a mechanistic link between obesity, insulin resistance and type 2 diabetes [13-15]. Local inflammation in VAT is associated with an infiltration of macrophages $[16,17]$, which are crucial for the production of pro-inflammatory cytokines and interfere with the effects of insulin on peripheral tissues via autocrine or paracrine signalling pathways $[18,19]$. The adipose tissue macrophages (ATMs) can be classified into a proinflammatory phenotype, termed M1 or 'classically activated macrophages', well correlated with insulin resistance [17, 20-22], and an anti-inflammatory phenotype, termed M2 or 'alternatively activated macrophages' $[16,17]$. While well established in mice [16, 17, 21, 22], the existence of M1 and M2 subsets of ATMs in humans has not been confirmed and human ATMs subtypes have rather been described with a mix of M1 and M2 gene expression profile [20]. Lymphocytes infiltrating adipose tissue might have a role in obesity-induced inflammation by modifying the number and activity of ATMs [23-25]. Particularly, regulatory T lymphocytes secrete antiinflammatory cytokines, such as IL-10, inhibit macrophage migration and induce the differentiation of M2-like macrophages $[19,21,23]$.

Notably, the elevated plasma levels of the pro-inflammatory cytokines IL-1 $\beta$ and IL-18 observed in obese people were found to predict the development of type 2 diabetes $[26,27]$. Active IL-1 $\beta$ and IL-18 are produced by the cleavage of their inactive precursors by the protease caspase- 1 , which is activated by large multiprotein complexes named inflammasomes [28]. Inflammasomes are central components of the innate immune system that respond to pathogen-associated (microbial products) and danger-associated (host-derived signals) molecular patterns. These patterns are recognised by intracellular NOD-like receptors (NLRs). The most extensively studied inflammasome is NLR family pyrin domain containing-3 (NLRP3). When activated, NLRP3 forms a complex with its adaptor molecule ASC, which in turn promotes the recruitment of pro-caspase 1 and its cleavage into active caspase-1 [28]. Compelling evidence shows that the NLRP3 inflammasome has a central role in obesity-induced inflammation and insulin resistance via these mechanisms [29-31].

The pathogenic effects of VAT might be related to the upregulated expression and activation of the NLRP3 inflammasome [32]. However, differences in NLRP3 inflammasome activity and immune cell composition in adipose tissue of MHO and MUO individuals remains largely unexplored. In this comparative study, we assessed whether the inflammatory profile of VAT was different in MUO individuals compared with that in MHO individuals and investigated the mechanisms that could contribute to such differences.

\section{Methods}

Participants and tissue sampling We studied 44 obese (BMI $>30 \mathrm{~kg} / \mathrm{m}^{2}$ ) patients (23 MUO and $21 \mathrm{MHO}$ patients) undergoing laparoscopic bariatric surgery and nine lean $\left(\mathrm{BMI}<25 \mathrm{~kg} / \mathrm{m}^{2}\right)$ individuals undergoing laparoscopic abdominal surgery (fundoplication for reflux disease or cholecystectomy for gallstone disease). The metabolic syndrome was defined in accordance with the commonly accepted definition [1]. Participants with three or more of the following criteria were classified as having the metabolic syndrome: (1) elevated waist circumference ( $\geq 94 \mathrm{~cm}$ in men and $\geq 80 \mathrm{~cm}$ in women); (2) elevated triacylglycerol level ( $\geq 1.7 \mathrm{mmol} / \mathrm{l})$; (3) reduced HDL-cholesterol level $(<1.0 \mathrm{mmol} / 1$ in men and $<1.3 \mathrm{mmol} / 1$ in women); (4) elevated BP (systolic BP $\geq 130 \mathrm{mmHg}$ and/or diastolic $\mathrm{BP} \geq 85 \mathrm{mmHg}$ ); (5) elevated fasting glucose level ( $\geq 5.6 \mathrm{mmol} / \mathrm{l})$. A metabolic syndrome score (from 0 to 5 ) was calculated as a function of the number of criteria shown by each patient. The MHO and MUO phenotypes were defined respectively as the absence (metabolic syndrome score $\leq 2$ ) or presence (metabolic syndrome score $>2$ ) of the metabolic syndrome. The MUO group comprised eight patients with type 2 diabetes, including four patients with non-treated newly diagnosed type 2 diabetes (with maximum $\mathrm{HbA}_{1 \mathrm{c}}$ of $6.6 \%$ [49 $\left.\mathrm{mmol} / \mathrm{mol}\right]$ ) and four patients with type 2 diabetes treated with insulin. Patients with inflammatory and malignant diseases or those treated with 
oral glucose-lowering drugs, such as metformin and sulfonylureas, were excluded. MHO and lean individuals had no history of cardiovascular, respiratory or metabolic diseases and did not receive any lipid-lowering, antihypertensive or glucose-lowering agents. The study protocol was approved by the ethics Committee of Liège University Hospital, and all patients provided written informed consent.

Metabolic variables were measured on fasting blood samples collected before the surgery. The degree of insulin resistance was estimated using the HOMA of insulin resistance (HOMA-IR) (fasting plasma glucose $[\mathrm{mmol} / \mathrm{l}] \times$ fasting plasma insulin pmol/l]/156).

Paired samples of SAT (abdominal wall) and VAT (greater omentum) were harvested during surgery and processed immediately. A portion of the sample was immediately frozen in liquid nitrogen and stored at $-80^{\circ} \mathrm{C}$. The remainder was gently washed in PBS and used directly for tissue culture or stromovascular cell (SVC) isolation.

Tissue culture Samples of SAT or VAT were individually placed in culture flasks with supplemented RPMI medium. Culture supernatant fractions were collected after $22 \mathrm{~h}$, concentrated and stored at $-20^{\circ} \mathrm{C}$ (see electronic supplementary material [ESM] Methods for further details).

Quantitative RT-PCR Total RNA was extracted from frozen adipose tissue using the RNeasy Lipid Tissue Mini Kit (Qiagen, Hilden, Germany), treated with DNase and reversetranscribed. Quantitative RT-PCR was performed using SYBER Green reagents in the ABI 7000 Sequence Detection System (Life Technologies, Carlsbad, CA, USA) or in the LightCycler 480 Real-Time PCR System (Roche, Basel, Switzerland) (see ESM Methods for further details). Results were normalised to HPRT1 and relative mRNA expression levels were calculated using $2^{-\Delta C_{t}}$ [33]. Primer sequences are provided in ESM Table 1.

SVC isolation and flow cytometry Adipose tissue samples were minced with sterile scissors and digested with type I collagenase (Sigma-Aldrich, St Louis, MO, USA). SVCs were isolated by successive centrifugations, washes and filtration (see ESM Methods for further details), and then were stained with saturating amounts of various fluorochromeconjugated antibodies from Ebioscience (Vienna, Austria) (CD45-phycoerythrin-cyanin 5 [PEC5], CD4-eFluor 450, CD25-phycoerythrin [PE], CD127 biotin and streptavidinallophycocyanin [APC]-eFluor 780) or BD Biosciences (San Jose, CA, USA) (CD3-Horizon v500, CD8-perdidin chlorophyll protein [PerCP] and CD14-FITC). Flow cytometry was performed on a FACSAria flow cytometer (BD Biosciences) and data were analysed using FacsDiva 6.1.2 (www. bdbiosciences.com/eu/instruments/software/facsdiva/) and FlowJo V.10 software (www.flowjo.com/download/). SVC subpopulations were determined based on sideward scatter and CD45 and CD14 expression. The ATMs were sorted from the $\mathrm{CD}_{4} 5^{+} \mathrm{CD} 14^{+} \mathrm{SVCs}$ with a purity of $>90 \%$, resuspended at a concentration of 50,000 cells in $500 \mu \mathrm{l}$ of supplemented RPMI medium and cultured at $37^{\circ} \mathrm{C}$. After $48 \mathrm{~h}$, the culture media were collected, centrifuged and stored at $-20^{\circ} \mathrm{C}$.

Caspase-1 activity assay SVCs were suspended in $75 \mu \mathrm{l}$ of $10 \mu \mathrm{mol} / \mathrm{l}$ fluorogenic caspase-1 substrate (CaspaLux E1-D2; Euromedex, Strasbourg, France) for $30 \mathrm{~min}$ at $37^{\circ} \mathrm{C}$. After washing in PBS supplemented with $3 \%$ FBS, cells were stained with combinations of antibodies from Ebioscience (CD45-PC5, CD4-eFluor 450) or BD Biosciences (CD14APC, CD14-PerCP, CD3-Horizon v500, CD8-PerCP, CD206-PE and CD11c-APC). Caspase-1 activity was measured in stained cells on a FACSCanto II flow cytometer (BD Biosciences) and analysed using FlowJo V.10 software.

ELISA IL-1 $\beta$ and IL-18 proteins were quantified in the culture media of ATMs or intact SAT and VAT by using cytokinespecific ELISA kits (R\&D Systems, Minneapolis, MN, USA) following the manufacturer's instructions.

Statistical analysis All results are expressed as the mean $\pm \mathrm{SE}$. GraphPad Prism software v.5 was used for statistical analysis (www.graphpad.com/scientific-software/prism/). Pairs of groups were compared by the Mann-Whitney $U$ test. Correlations were assessed using a Spearman correlation test. A $p$ value $<0.05$ was considered statistically significant.

\section{Results}

Anthropometric, clinical and biological characteristics of the study population Clinical and biological details of the patients are summarised in Table 1. Metabolic variables were similar among MHO patients and healthy lean participants, as was arterial BP. Body weight and BMI were similar in the MUO and MHO group; however, MUO patients had a significantly increased waist circumference compared with $\mathrm{MHO}$ patients. Both obese groups had significantly higher levels of C-reactive protein than the lean group had. Compared with the MHO group, the MUO group had increased levels of alanine and aspartate transaminases.

The VAT of MUO patients is characterised by increased IL-1 $\beta$ production compared with that of MHO patients IL-1 $\beta$ and IL-18 levels were measured in the SAT and VAT of each study group. Relative to the corresponding SAT, the production of IL-1 $\beta$ protein was significantly increased in the VAT of MUO patients (Fig. 1a), whereas IL-18 production was increased in 
Table 1 Anthropometric, clinical and biological characteristics of the participants
Values are means $\pm \mathrm{SE}$

The metabolic syndrome is defined as three or more of the following: waist $\geq 80 \mathrm{~cm}$ in women and $\geq 94 \mathrm{~cm}$ in men, BP $\geq 130 / 85 \mathrm{mmHg}$, fasting glucose $\geq 5.6 \mathrm{mmol} / 1$, HDL-cholesterol $<1.3 \mathrm{mmol} / \mathrm{l}$ in women and 1.0 in men and triacylglycerols $>1.7 \mathrm{mmol} / 1$

${ }^{*} p<0.05$ vs lean; ${ }^{\dagger} p<0.05$ vs $\mathrm{MHO}$

\begin{tabular}{|c|c|c|c|}
\hline Characteristic & Lean & MHO & MUO \\
\hline$n$ (male/female sex) & $9(5 / 4)$ & $21(3 / 18)$ & $23(7 / 16)$ \\
\hline Age (years) & $42 \pm 4$ & $35 \pm 2$ & $42 \pm 3$ \\
\hline Body weight (kg) & $64.1 \pm 4$ & $121 \pm 4.2 *$ & $123.5 \pm 5.4^{*}$ \\
\hline BMI $\left(\mathrm{kg} / \mathrm{m}^{2}\right)$ & $22.7 \pm 0.9$ & $42.3 \pm 1 *$ & $42.7 \pm 1.1^{*}$ \\
\hline Waist circumference $(\mathrm{cm})$ & $73 \pm 2.8$ & $115.7 \pm 2 *$ & $128.7 \pm 2.7^{* \dagger}$ \\
\hline Systolic BP (mmHg) & $115.1 \pm 7.6$ & $123.1 \pm 3$ & $135.8 \pm 3 *, \dagger$ \\
\hline Diastolic BP (mmHg) & $68.1 \pm 5.5$ & $75.5 \pm 2$ & $87.1 \pm 2^{*, \dagger}$ \\
\hline Fasting glucose $(\mathrm{mmol} / \mathrm{l})$ & $4.9 \pm 0.1$ & $4.9 \pm 0.1$ & $6.2 \pm 0.3^{*, \dagger}$ \\
\hline Fasting insulin (pmol/l) & $68.40 \pm 9.0$ & $116.12 \pm 15.9$ & $195.6 \pm 30.6^{*}$ \\
\hline HOMA-IR & $2.15 \pm 0.27$ & $3.65 \pm 0.43$ & $7.77 \pm 1.78^{* \dagger}$ \\
\hline \multicolumn{4}{|l|}{$\mathrm{HbA}_{1 \mathrm{c}}$} \\
\hline $\mathrm{HbA}_{1 \mathrm{c}}(\%)$ & $5.6 \pm 0.07$ & $5.5 \pm 0.06$ & $6.2 \pm 0.23^{*, \dagger}$ \\
\hline $\mathrm{HbA}_{1 \mathrm{c}}(\mathrm{mmol} / \mathrm{mol})$ & $37.6 \pm 0.69$ & $37.1 \pm 0.70$ & $43.8 \pm 2.49^{*, \dagger}$ \\
\hline Triacylglycerols (mmol/l) & $1.1 \pm 0.2$ & $1.2 \pm 0.1$ & $1.8 \pm 0.2^{*, \dagger}$ \\
\hline Total cholesterol $(\mathrm{mmol} / \mathrm{l})$ & $4.2 \pm 0.5$ & $4.5 \pm 0.2$ & $4.8 \pm 0.2$ \\
\hline HDL-cholesterol (mmol/l) & $1.1 \pm 0.2$ & $1.2 \pm 0.1$ & $1.1 \pm 0.1$ \\
\hline LDL-cholesterol $(\mathrm{mmol} / \mathrm{l})$ & $2.3 \pm 0.3$ & $2.6 \pm 0.2$ & $2.8 \pm 0.2$ \\
\hline Alanine transaminase $(\mu \mathrm{kat} / \mathrm{l})$ & $0.46 \pm 0.1$ & $0.40 \pm 0.1$ & $0.61 \pm 0.1^{\dagger}$ \\
\hline Aspartate transaminase $(\mu \mathrm{kat} / \mathrm{l})$ & $0.39 \pm 0.1$ & $0.32 \pm 0.1$ & $0.43 \pm 0.1^{\dagger}$ \\
\hline$\gamma$-Glutamyl transpeptidase $(\mu \mathrm{kat} / \mathrm{l})$ & $0.40 \pm 0.1$ & $0.39 \pm 0.1$ & $0.57 \pm 0.1$ \\
\hline C-reactive protein $(\mathrm{nmol} / \mathrm{l})$ & $17.4 \pm 6.7$ & $63.8 \pm 7.6^{*}$ & $80.9 \pm 9.5^{*}$ \\
\hline Type 2 diabetes $(n)$ & $0 / 9$ & $0 / 21$ & $8 / 23$ \\
\hline Metabolic syndrome score & $0.9 \pm 0.3$ & $1.9 \pm 0.1 *$ & $3.7 \pm 0.2 *, \dagger$ \\
\hline
\end{tabular}

the VAT of both obese groups (Fig. 1b). The levels of both cytokines were significantly increased in the VAT of MUO patients compared with those in the VAT of lean patients (Fig. 1a, b). IL-1 $\beta$ production was increased fourfold in the VAT of MUO patients compared with the VAT of MHO patients (Fig. 1a).

The expression of IL-1 $\beta$ and NLRP3 is upregulated in the VAT of MUO patients and correlates with the metabolic syndrome and insulin resistance The production of activated IL-1 $\beta$ depends on two signals. First, a pro-inflammatory signal stimulates the transcription of ILIB [28]. A second signal then induces inflammasome formation and allows caspase- 1 activation and subsequent cleavage of the inactive pro-IL- $1 \beta$ into active cytokine. In contrast to IL- $1 \beta$, the regulation of IL-18 biological activity is mainly due to caspase- 1 mediated proIL-18 processing [34]. To investigate whether the observed differences in IL-1 $\beta$ levels were related to differences in the first signal, ILIB mRNA levels were measured by quantitative RT-PCR analysis of total RNA samples purified from SAT and VAT. IL18 expression was also determined in these samples.

The observed trends in $I L 1 B$ expression were similar to those for cytokine production. The $I L 1 B \mathrm{mRNA}$ level did not change in the SAT but increased in the VAT of both MUO and
MHO patients when compared with the tissues of lean participants (Fig. 1c). A significant increase in $I L 1 B$ expression was found in the VAT of MUO patients compared with that of MHO patients (Fig. 1c). IL18 mRNA levels were much lower in the SAT relative to the VAT, regardless of the patient's phenotype (Fig. 1d) and did not correlate with the protein levels detected by ELISA. Indeed, IL-18 production was increased in the MUO group compared with that in lean patients (Fig. 1b) despite a decreased IL18 gene expression (Fig. 1d).

In accordance with these data, we found, in the overall study population, significant correlations between $I L 1 B$ or IL18 expression in VAT and various patient's characteristics (Table 2), whereas no significant correlation was found between the expression of these genes in SAT and characteristics of the patients (data not shown).

NLRP3 expression was increased in the VAT of MUO patients compared with that of lean and MHO patients, whereas no difference was observed between lean and MHO participants (Fig. 2a). Moreover, in the MUO group, NLRP3 mRNA levels were twofold higher in VAT than in SAT (Fig. 2a). In the overall study population, positive correlations between the NLRP3 expression in VAT and patient's characteristics are shown in Table 2. ASC (also known as PYCARD) and CASP1 
a
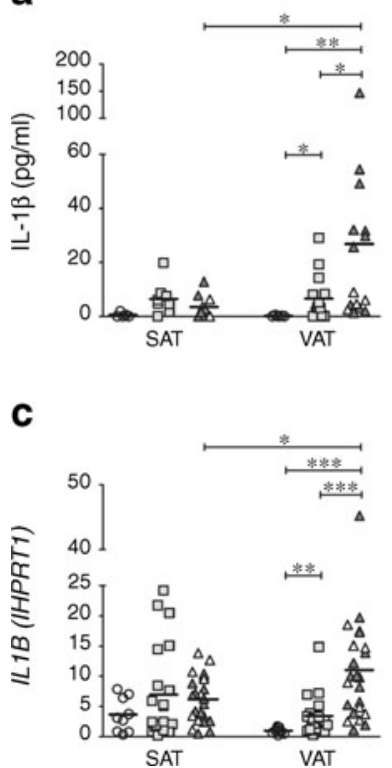

b

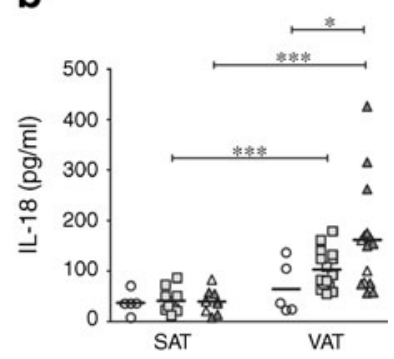

d

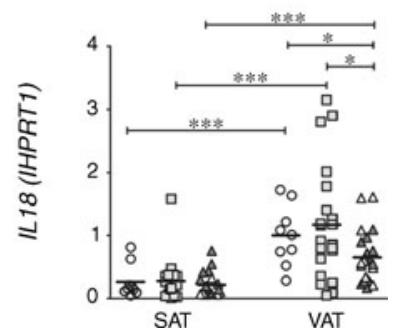

Fig. 1 IL-1 $\beta$ and IL-18 protein production and gene expression in the SAT and VAT of lean, MHO and MUO participants. (a) IL-1 $\beta$ production from the SAT and VAT of lean (white circles), MHO (white squares) and MUO (grey triangles) $(n=5,15$ and 15 [including 4 with type 2 diabetes, white triangles], respectively). (b) IL-18 production from the SAT and VAT of lean (white circles), MHO (white squares) and MUO (grey triangles) patients ( $n=5,15$ and 15 [including 4 with type 2 diabetes, white triangles], respectively). Intact adipose tissue fragments were cultured for $22 \mathrm{~h}$ and cytokine levels were measured in the supernatant fraction by ELISA. Relative $I L 1 B$ (c) and IL18 (d) mRNA expression in the whole SAT and VAT of lean (white circles), MHO (white squares) and MUO (grey triangles) ( $n=9,21,23$ [including 8 with type 2 diabetes, white triangles], respectively). Total RNA was extracted, and mRNA levels were measured by quantitative RT-PCR. Data were normalised according to HPRT1 mRNA levels and presented as a relative value to that for the VAT of lean participants. The horizontal lines among the data points represent the means. ${ }^{*} p<0.05,{ }^{* *} p<0.01,{ }^{* * *} p<0.001$ (MannWhitney $U$ test)

expression was not significantly affected by obesity or the metabolic syndrome either in SAT or in VAT (Fig. 2b, c). Other inflammasomes (NLRP1, NLRC4 and AIM2) were also investigated. In each study group, NLRP1 mRNA levels were lower in VAT than in SAT (Fig. 2d). Expression patterns of NLRC4 and AIM2 were similar in the SAT and VAT of each group (Fig. 2e, f). The expression of two other NLRs, NOD1 and NOD2, which are not involved in the inflammasome pathway but might play a role in the pathophysiology of type 2 diabetes $[35,36]$, was also similar in the SAT and VAT of lean and obese participants (ESM Fig. 1).

In summary, IL-1 $\beta$ production in VAT was increased among MUO patients (Fig. 1a) and was correlated to an upregulated transcription of IL1B (Fig. 1c) and NLRP3 (Fig. 2a).

The metabolic syndrome is associated with an increased number of ATMs and a decreased number of regulatory $T$ cells in VAT As inflammatory cytokines are produced by macrophages and their production is inhibited by regulatory $\mathrm{T}$
Table 2 Correlations between characteristics of the study population and $I L 1 B, I L 18$ and $N L R P 3$ gene expression in VAT

\begin{tabular}{llll}
\hline Characteristic & $\begin{array}{l}I L 1 B \\
\text { mRNA }\end{array}$ & $\begin{array}{l}\text { IL18 } \\
\text { mRNA }\end{array}$ & $\begin{array}{l}\text { NLRP3 } \\
\text { mRNA }\end{array}$ \\
\hline BMI & $0.39^{*}$ & -0.09 & 0.2 \\
Waist circumference & $0.5^{* *}$ & -0.20 & $0.5^{* *}$ \\
Metabolic syndrome score & $0.57^{* * * *}$ & $-0.47^{* *}$ & $0.39^{*}$ \\
Systolic BP & $0.33^{*}$ & -0.09 & 0.12 \\
Diastolic BP & $0.39^{*}$ & -0.16 & 0.19 \\
Fasting glucose & $0.56^{* * * *}$ & -0.27 & $0.35^{*}$ \\
HOMA-IR & $0.53^{* *}$ & $-0.46^{*}$ & $0.65^{* * * *}$ \\
HbA $1 \mathrm{c}$ & 0.02 & $-0.32^{*}$ & 0.12 \\
Triacylglycerols & 0.09 & $-0.30^{*}$ & 0.11 \\
HDL-cholesterol & -0.19 & 0.04 & -0.15 \\
LDL-cholesterol & 0.17 & -0.03 & 0.02 \\
C-reactive protein & $0.42^{*}$ & -0.19 & $0.44^{*}$ \\
\hline
\end{tabular}

Correlations were assessed using a Spearman correlation test

$* p<0.05, * * p<0.001$ and $* * * * p<0.0001$

cells $[19,21,23]$, we hypothesised that the increased $I L 1 B$ and NLRP3 expression in the VAT of MUO patients reflected variations in the composition of the SVCs. To investigate this assumption, flow cytometry analyses were performed on SVCs isolated from SAT and VAT. Anti-CD45 and antiCD14 antibodies were used to differentiate ATMs from the granulocytes, lymphocytes and pre-adipocytes. The number of these cell types was expressed as a percentage of the total SVCs (Table 3). The percentage of ATMs was significantly increased in the VAT of MUO participants compared with either their SAT or the VAT of MHO and lean participants, whereas no difference was found between the MHO and the lean groups. The same pattern was observed for granulocytes. Unlike ATMs and granulocytes, the percentage of preadipocytes decreased in the VAT of patients with metabolic abnormalities. Similarly, pre-adipocytes were less numerous in the VAT than in the SAT of MUO patients. The percentage of lymphocytes was higher in the VAT compared with the SAT in both groups of obese patients.

Subsequent flow cytometry analyses on adipose tissuederived SVCs were performed to assess the composition of lymphocyte subpopulations (Table 3). An increased T lymphocyte population was observed in the VAT vs the SAT of obese patients. The percentage of $\mathrm{CD} 8^{+} \mathrm{T}$ cells was significantly increased in the VAT of all three study groups compared with their SAT, whereas the proportion of $\mathrm{CD}^{+} \mathrm{T}$ cells did not differ (Table 4). No differences were found between the MHO and MUO groups in the composition of these T cell populations. Interestingly, however, a significant reduction in regulatory $\mathrm{CD}^{+} \mathrm{T}$ cell percentage was observed in the VAT of MUO patients compared with either the corresponding SAT or the VAT of MHO and lean participants (Fig. 3a, b). 
a

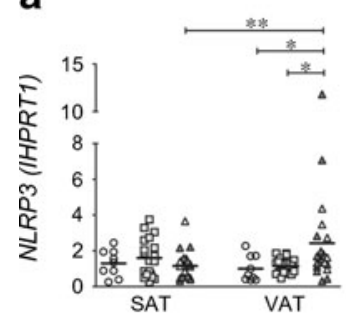

d

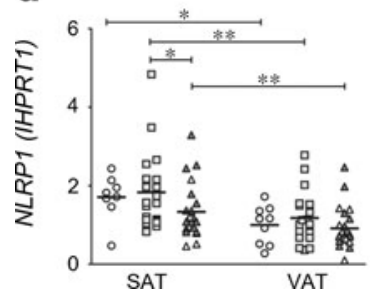

b

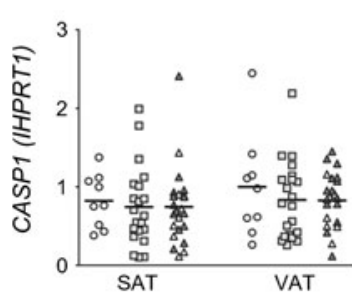

e

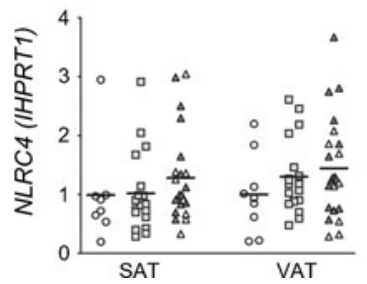

C

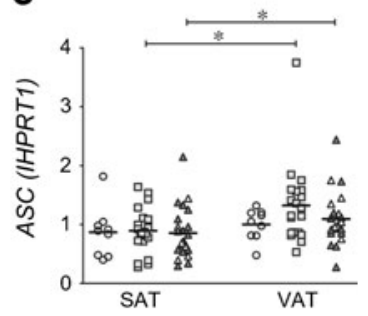

f

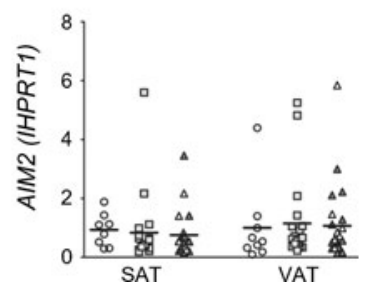

Fig. 2 Expression of inflammasome components in the SAT and VAT of lean, $\mathrm{MHO}$ and MUO patients. Relative gene expression of $N L R P 3$ (a), CASP-1 (b), ASC (c), NLRP1 (d), NLRC4 (e) and AIM2 (f) in the SAT and VAT of lean (white circles), MHO (white squares) and MUO (grey triangles) patients $(n=9,21$ and 23 [including 8 with type 2 diabetes, white triangles], respectively). Total RNA was extracted from the whole adipose tissue fragment, and quantitative RT-PCR was performed as

These results demonstrate that the VAT of MUO patients is characterised by an ATM and granulocyte infiltration and a decreased percentage of anti-inflammatory regulatory $\mathrm{T}$ cells.

The metabolic syndrome is associated with inflammasome activation in ATMs isolated from VAT To assess the potential contribution of immune cells to adipose tissue inflammation, we examined caspase-1 activity in each subpopulation. SVCs were incubated with a fluorogenic caspase-1 substrate and stained for flow cytometry analyses. Subsequently, caspase-1 activity was evaluated with gating on different subpopulations of SVCs (Fig. 4a-c).

Caspase-1 activity was very low in lymphocytes (Fig. 4a) and in granulocytes (Fig. 4b) and was not significantly different between lean, MHO and MUO individuals, either in SAT or in VAT (data not shown). As expected, caspase-1 activity was elevated in ATMs but the increase was not uniform throughout the ATM population (Fig. 4c). Indeed, two subsets of ATMs could be discerned among lean and MHO patients on the basis of their caspase-1 activity level (Fig. 4c); these subsets were described in Methods. Data were normalised according to HPRT1 mRNA levels and presented as a value relative to that for the VAT of lean participants. The horizontal lines among the data points represent the means. ${ }^{*} p<0.05,{ }^{*} p<0.01,{ }^{* * *} p<0.001$ (Mann-Whitney $U$ test). The exclusion of the two highest values for NLRP3 expression in the VAT of MUO does not change the statistical observations

similarly distributed in the two groups (Fig. 4d). Interestingly, the proportion of ATMs with high caspase-1 activity was significantly increased in the VAT of MUO patients (Fig. 4d). By contrast, no differences were observed between the three patient groups in the distribution of ATMs in SAT (Fig. 4d).

To further confirm the differences in caspase- 1 activity observed between MHO and MUO patients, the level of human IL- $1 \beta$ was measured by ELISA in the culture media of ATMs isolated from the VAT of MHO and MUO patients. As expected, ATMs isolated from the VAT of MUO patients produced significantly greater amounts of IL- $1 \beta$ than those isolated from the VAT of MHO patients (Fig. 4e).

In conclusion, the development of the metabolic syndrome in obese individuals is associated with inflammasome activation in ATMs infiltrating VAT, which leads to increases in both caspase-1 activity and IL-1 $\beta$ secretion.

Activation of caspase-1 is increased in $C D 11 c^{+} C D 206^{+}$ ATMs Previous studies have shown that ATMs comprise $\mathrm{CD} 11 \mathrm{c}^{+} \mathrm{CD} 206^{+}$cells and $\mathrm{CD} 11 \mathrm{c}^{-} \mathrm{CD} 206^{+}$cells [20].
Table 3 Composition of SVCs in the adipose tissue

Data are presented as \% of SVCs $($ mean $\pm \mathrm{SE})$

${ }^{*} p<0.05$ vs lean (within same tissue) ${ }^{\dagger} p<0.05$ vs MHO (within same tissue); ${ }^{\star} p<0.05$ vs SAT (within same phenotype)

\begin{tabular}{|c|c|c|c|c|c|c|}
\hline \multirow[t]{2}{*}{ SVC composition } & \multicolumn{3}{|l|}{ SAT } & \multicolumn{2}{|l|}{ VAT } & \multirow[b]{2}{*}{ MUO } \\
\hline & Lean & MHO & MUO & Lean & MHO & \\
\hline Macrophages & $5.5 \pm 1.8$ & $7.5 \pm 0.9$ & $7.0 \pm 1.1$ & $6.1 \pm 1.1$ & $7.9 \pm 0.7$ & $10.8 \pm 1.2^{* \dagger \dagger, \dagger}$ \\
\hline Granulocytes & $7.8 \pm 3.7$ & $7.8 \pm 2.0$ & $7.6 \pm 1.5$ & $3.8 \pm 1.1$ & $6.4 \pm 1.6$ & $13.0 \pm 2.2 *, \dagger,+$ \\
\hline Pre-adipocytes & $82.0 \pm 6.4$ & $78.5 \pm 2.7$ & $80.6 \pm 2.7$ & $82.6 \pm 2.9$ & $74.4 \pm 2.3$ & $65.6 \pm 2.9^{*, \dagger, \dagger}$ \\
\hline Lymphocytes & $4.7 \pm 1.6$ & $6.1 \pm 0.9$ & $5.4 \pm 0.9$ & $8.0 \pm 1.9$ & $11.3 \pm 1.1^{\ddagger}$ & $11.7 \pm 1.1^{\ddagger}$ \\
\hline
\end{tabular}


Table 4 Composition of the lymphocyte subsets in the adipose tissue

Data are means $\pm \mathrm{SE}$

${ }^{*} p<0.05$ vs SAT within same phenotype

\begin{tabular}{|c|c|c|c|c|c|c|}
\hline \multirow[t]{2}{*}{ Lymphocyte composition } & \multicolumn{3}{|l|}{ SAT } & \multicolumn{3}{|l|}{ VAT } \\
\hline & Lean & MHO & MUO & Lean & MHO & MUO \\
\hline T cells ( $\%$ of SVCs) & $2.6 \pm 1.0$ & $4.4 \pm 0.7$ & $4.3 \pm 0.8$ & $6.3 \pm 2.3$ & $9.6 \pm 1.2 *$ & $9.9 \pm 0.9^{*}$ \\
\hline CD4 T cells ( $\%$ of T cells) & $58.9 \pm 5.0$ & $56.1 \pm 2.0$ & $49.9 \pm 2.7$ & $45.7 \pm 4.8$ & $50.3 \pm 2.0$ & $48.2 \pm 2.1$ \\
\hline CD8 T cells ( $\%$ of T cells) & $25.6 \pm 3.3$ & $26.5 \pm 1.9$ & $30.2 \pm 2.0$ & $41.3 \pm 2.8^{*}$ & $36.1 \pm 2.1 *$ & $36.3 \pm 2.3^{*}$ \\
\hline
\end{tabular}

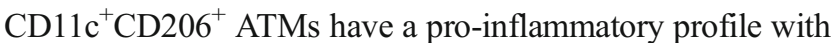
an M1 phenotype, are markers of insulin resistance in human obesity [20, 37] and secrete more IL-1 $\beta$ than do CD11 ${ }^{-}$CD206 ${ }^{+}$ATMs [20]. As we observed two subsets of ATMs with different caspase-1 activity levels (Fig. 4c), we wondered whether these ATM subpopulations corresponded

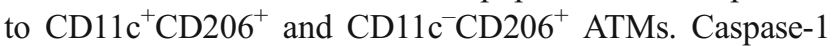
activity was measured in $\mathrm{CD} 14^{+} \mathrm{CD} 11 \mathrm{c}^{+} \mathrm{CD} 206^{+}$and CD $14^{+}$CD $11 \mathrm{c}^{-} \mathrm{CD} 206^{+}$ATMs. Regardless of a patient's body weight and metabolic abnormalities and the selected adipose tissue, ATMs with high caspase-1 activity were mainly

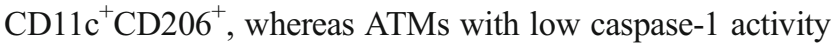
were mainly CD11c ${ }^{-}$CD206 ${ }^{+}$(Fig. 5a). Likewise, we showed that caspase-1 activity was significantly increased in CD $11 \mathrm{c}^{+} \mathrm{CD}_{206} \mathrm{C}^{+} \mathrm{ATMs}$ compared with that in CD11c ${ }^{-}$CD206 ${ }^{+}$ATMs in both SAT and VAT (Fig. 5b).

\section{Discussion}

This study is the first to compare NLRP3 inflammasome activity involved in obesity-induced inflammation and insulin resistance [29-32] and the composition of the SVCs in paired SAT and VAT samples taken from obese patients with or without the metabolic syndrome. We describe significant differences in the inflammatory profile of VAT from MUO patients compared with that from MHO patients. First, NLRP3 and ILIB expression was upregulated in the VAT of MUO patients compared with that of lean and MHO patients, providing indirect evidence for the role of the NLRP3 inflammasome in the heterogeneity of obesity. Second, the VAT of MUO patients was mainly infiltrated by proinflammatory macrophages with an increased caspase-1 activity and thus an increased IL- $1 \beta$ release, whereas the VAT of MHO and lean individuals was infiltrated with fewer macrophages and these macrophages had a lower caspase-1 activity. The macrophages with higher and lower caspase-1 activity were found to correspond mainly to $\mathrm{CD} 11 \mathrm{c}^{+} \mathrm{CD} 206^{+}$and CD11 $\mathrm{C}^{-} \mathrm{CD} 206^{+}$ATMs, respectively. Finally, the percentage of anti-inflammatory regulatory $\mathrm{T}$ cells was decreased in the VAT of MUO patients compared with MHO patients. Similar differences were observed between the VAT and the SAT of MUO patients. Changes in NLRP3 and $I L 1 B$ expression, inflammasome activity and SVCs were not observed in SAT samples between the three phenotypes.

So the increase in IL-1 $\beta$ production by the VAT of MUO patients could be explained by a higher number of total ATMs and an increased part of ATMs with a higher caspase-1 a

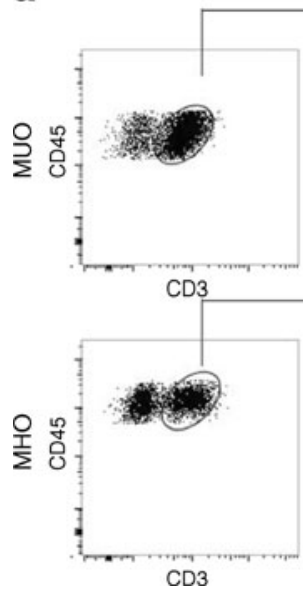

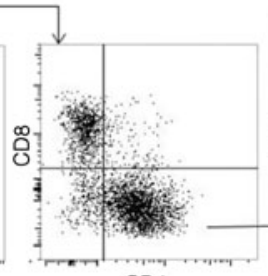

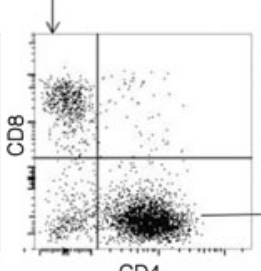

CD4

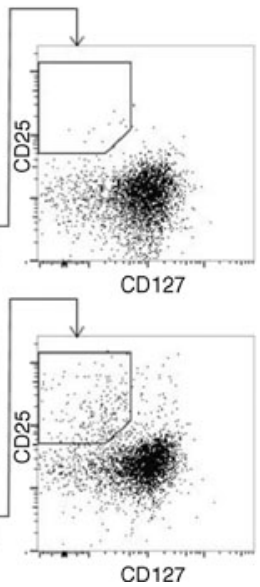

MHO (white squares) and MUO (grey triangles) patients $(n=7,15,19$ [including 8 with type 2 diabetes (white triangles)], respectively). The horizontal lines among the data points represent the means. ${ }^{*} p<0.05$, $* * p<0.01$ (Mann-Whitney $U$ test)

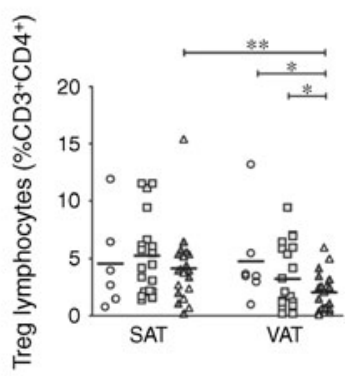

Fig. 3 Decreased number of regulatory T cells in the VAT of MUO patients. (a) Representative FACS plots showing $\mathrm{CD}^{+} \mathrm{CD} 4^{+}$ $\mathrm{CD} 25^{+} \mathrm{CD} 127^{\text {low }}$ regulatory $\mathrm{T}$ cells from the VAT of MHO and MUO patients. (b) Gated percentages of regulatory T cells (Treg) among the $\mathrm{CD}^{+} \mathrm{T}$ cell population from the SAT and VAT of lean (white circles),

b 
a

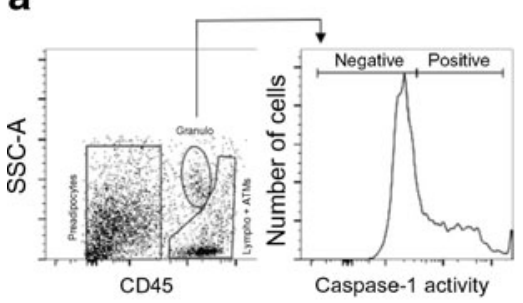

b

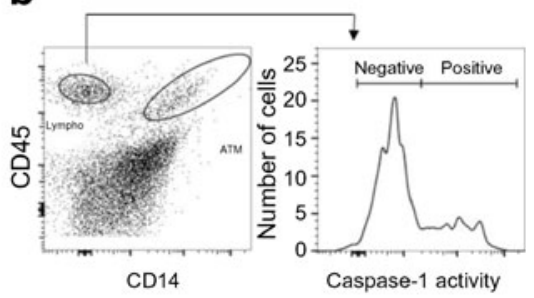

C

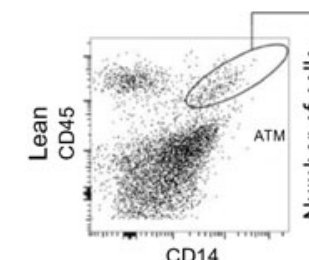

CD14

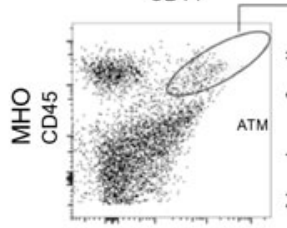

CD14

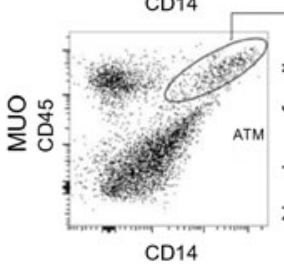

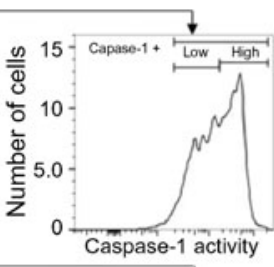
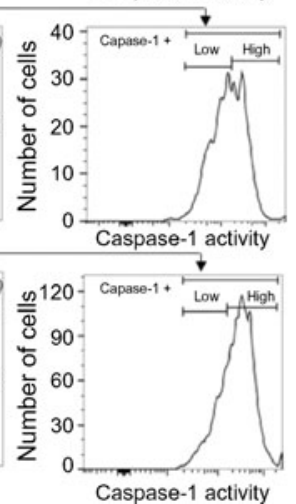

d

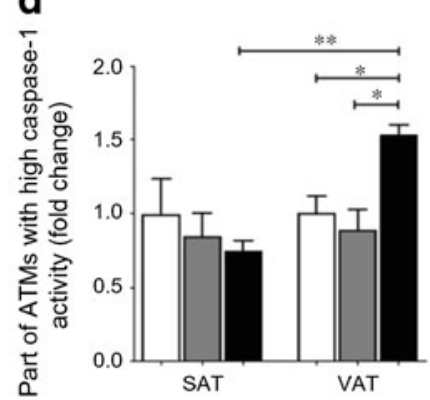

e

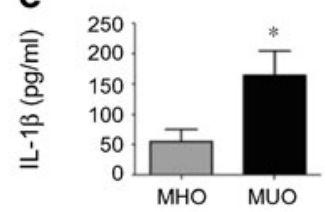

Fig. 4 Increased inflammasome activity in ATMs from the VAT of MUO patients. Representative FACS data of caspase-1 activity. Caspase-1 activity was evaluated by gating (a) on the granulocyte population differentiated from the other cells with CD45 staining and sideward scatter, (b) on the lymphocyte population differentiated from the other cells with CD14 and CD45 staining and (c) on the ATM population differentiated from other cells with CD14 and CD45 staining and extracted from the VAT of lean, MHO and MUO patients. In the lean and MHO groups, we distinguished two levels of caspase-1 activity, which indicates that a fraction of the ATMs have lower caspase-1 activity

activity. In addition, previous studies have shown that insulin resistance is associated with an increased pro-inflammatory $\mathrm{CD} 11 \mathrm{c}^{+} \mathrm{CD} 206^{+}$adipose tissue density and $\mathrm{CD} 11 \mathrm{c}^{+} \mathrm{CD} 206^{+} /$

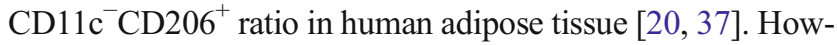
ever, because obesity and insulin resistance are also associated with increased expression and production of other proinflammatory cytokines, such as TNF- $\alpha$ and IL-6, in adipose tissue $[14,38,39]$, this observed increase in IL- $1 \beta$ production could be also due to a local inflammation in the VAT of MUO patients.

As previously observed [40-42], we found a lower IL18 expression in the VAT of MUO patients compared with MHO and lean participants, whereas their IL-18 secretion was upregulated. Unlike the expression of $I L 1 B$, the expression of $I L 18$ is constitutive and not modulated by proinflammatory conditions [34] and the decrease in IL18 mRNA than the others. (d) The parts of gated ATMs with higher caspase-1 activity in the SAT and VAT of lean (white bars), MHO (grey bars) and MUO (black bars) participants $(n=4,9,5$ [including 1 with type 2 diabetes], respectively). Data are presented as a value relative to that for the VAT of lean individuals. (e) IL- $1 \beta$ production by the ATMs $(50,000$ cells/well) isolated from the VAT of MHO $(n=6)$ and MUO $(n=9$, including 2 with type 2 diabetes) patients and cultured for $48 \mathrm{~h}$. The results are shown as the mean $\pm \mathrm{SE} .{ }^{*} p<0.05,{ }^{* *} p<0.01$ (Mann-Whitney $U$ test)

levels in the VAT of MUO patients might reflect differences in cell composition.

In this study, we did not directly measure visceral fat mass; only waist circumference was measured, which in itself cannot discriminate between subcutaneous and visceral obesity. However, an increased waist circumference combined with elevated levels of plasma triacylglycerols (a condition called 'hypertriacylglycerolaemic waist') is known to be associated with a very high probability of visceral obesity [43]. Among MUO participants enrolled in this study, hypertriacylglycerolaemic waist was indeed a potent indicator of excess visceral fat mass. By contrast, MHO patients had normal plasma triacylglycerol levels and, independently of their body weight and BMI, had a smaller waist circumference than MUO patients. Thus, a relative increase in the amount of VAT could explain the less favourable metabolic profile found 
Fig. 5 Caspase-1 activity in $\mathrm{CD} 11 \mathrm{c}^{+} \mathrm{CD} 206^{+}$and CD11 $\mathrm{c}^{-} \mathrm{CD} 206^{+}$ATMs from SAT and VAT. (a) Representative FACS data of ATMs from VAT of lean, MHO and MUO patients, with high or low caspase-1 activity, stained with CD206 and CD11c. After adipose tissue digestion, SVCs were incubated for $30 \mathrm{~min}$ with fluorogenic caspase- 1 substrate and stained for FACS analysis with conjugated CD45, CD14, CD11c and CD206, as described in Methods. Caspase-1 activity in ATMs was evaluated by gating on the ATM population differentiated from other cells with CD14 and CD45 staining. ATMs with high caspase-1 activity were mainly $\mathrm{CD}_{11 \mathrm{c}^{+} \mathrm{CD} 206^{+} \text {, whereas ATMs }}$ with lower caspase-1 activity were mainly CD $11 \mathrm{c}^{-} \mathrm{CD} 206^{+}$. (b) Differences in caspase-1 activity, evaluated by median intensity fluorescence (MFI), in

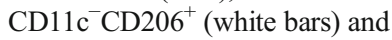

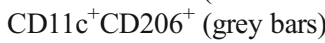
ATMs from the SAT and VAT of all study participants combined ( $n=11$, including 4 lean, $4 \mathrm{MHO}$ and 3 MUO patients). The results are shown as the mean $\pm \mathrm{SE}$. ${ }^{*} p<0.05$ (Mann-Whitney $U$ test) (c) Unstained control ATMs for CD11c-APC and CD206-PE
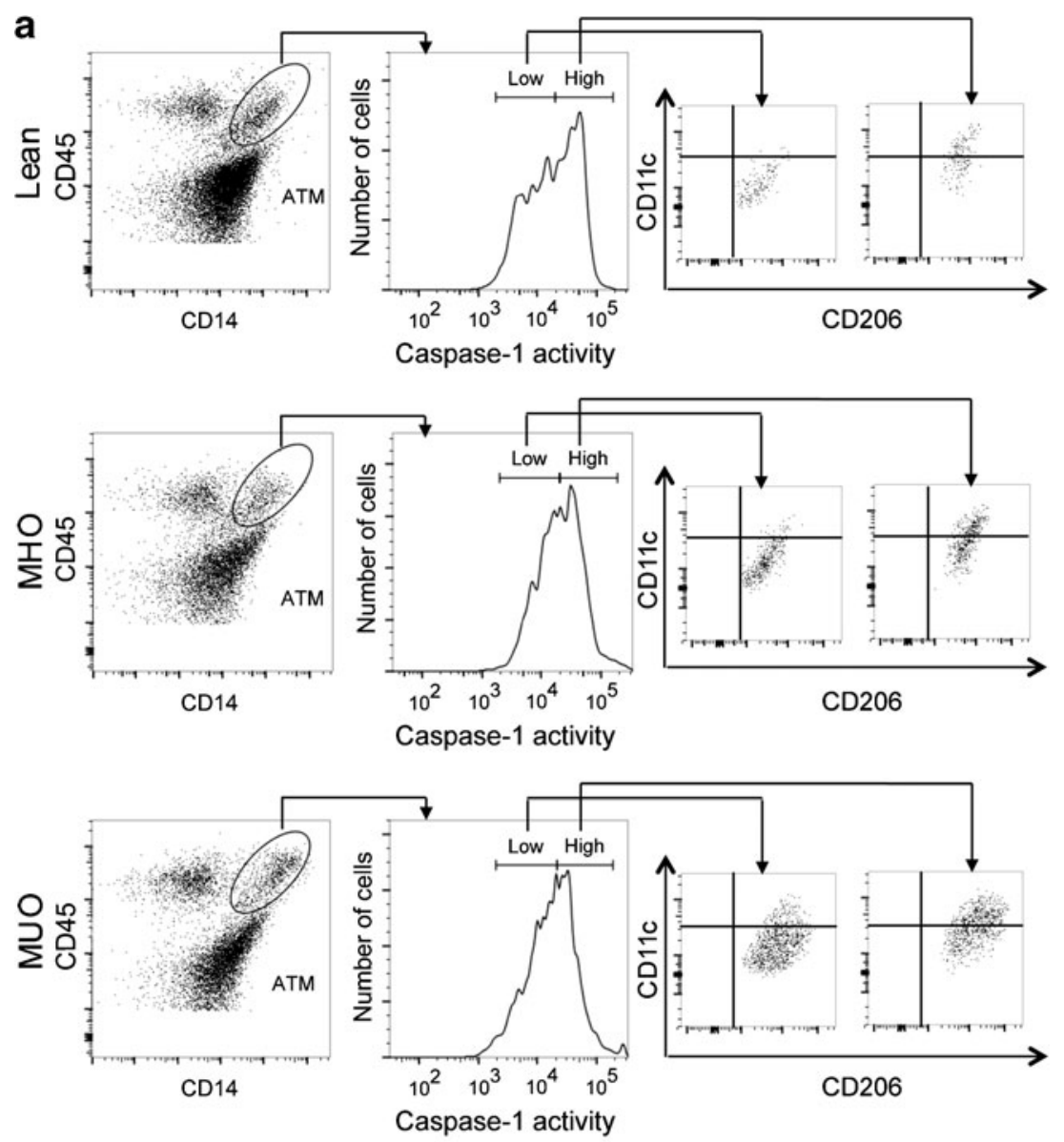

b

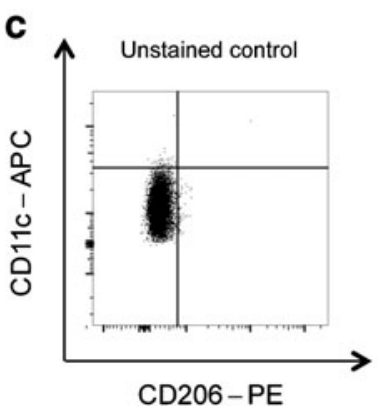

among MUO patients. Moreover, we found consistent positive correlations between waist circumference and the NLRP3 and $I L 1 B$ gene expression in VAT in the overall study population. Some of the findings with regard to the inflammasome also positively correlate with the metabolic syndrome score, fasting glucose, HOMA-IR and BP in the whole cohort of participants. Further research should also be aimed at investigating the immunological profile and inflammasome activity in other anatomical fat localisations such as the deeper subcutaneous, mesenteric and retroperitoneal adipose tissues.

The nature of the stimuli that trigger inflammasome activity and caspase- 1 activation in ATMs, and thereby may cause the observed differences between MHO and MUO individuals, remains to be determined. Saturated fatty acids can induce inflammatory cascades in macrophages and adipocytes through Toll-like receptor 4-dependent effects [44] and through activation of the NLRP3 inflammasome [45]. In contrast, unsaturated fatty acids exert strong antiinflammatory effects resulting in improved insulin sensitivity in diet-induced obese mice [46] and do not activate the Tolllike receptor 4 or the NLRP3 inflammasome [45]. Therefore, future studies must determine whether fat composition of diets could partially explain the differences observed in this study.

$\mathrm{T}$ lymphocytes accumulate in adipose tissue before macrophage populations do and may contribute to metabolic abnormalities by controlling their activity [23, 25, 47]. Consistently, we showed that the number of $\mathrm{T}$ lymphocytes, particularly cytotoxic $\mathrm{CD}^{+}$cells, was increased in the VAT compared 
with the SAT in all three patient groups. However, we did not find any difference between the patient groups in the number of these cells. Furthermore, the percentage of regulatory T cells was decreased in the VAT of MUO patients compared with the corresponding SAT and with the VAT of MHO and lean participants. Previous studies showed that the number of regulatory $\mathrm{T}$ cells in VAT decreases with obesity in both mice and humans $[23,25,37]$ and that a boost in the number of these cells can improve insulin sensitivity in diet-induced obese mice [23]. These cells also induce differentiation of M2-like macrophages [19, 21, 23]. Therefore, T regulatory cells may repress adipose tissue inflammation in MHO and lean individuals. As it has been shown that immunotherapy using a CD3-specific antibody ameliorates obesity-associated insulin resistance [25], identifying the mechanisms that lead to the decrease in regulatory T cells in the VAT of MUO patients might offer therapeutic options [47].

This study has some limitations. First, even though the number of patients included in the various groups allows us to observe statistical differences, it remains rather limited. Second, the MUO study group represents a quite heterogeneous population including patients with the metabolic syndrome, patients with non-treated type 2 diabetes and patients with insulin-treated type 2 diabetes. Nevertheless, there was no significant change in the results when insulin-treated diabetic patients were excluded from the MUO group (data not shown). Third, MHO and MUO phenotypes were defined based on the absence or presence of the metabolic syndrome. However, various definitions of MHO and MUO have been used throughout the literature and there is no standardised definition of these phenotypes yet $[3,4]$. Fourth, the MHO and MUO groups had not been matched for the sex distribution. However, there is no difference in gene expression profile, interleukin production and SVC composition in the VAT between obese men and women, regardless of our classification based on the metabolic syndrome score (data not shown). Finally, a precise assessment of fat distribution (e.g. quantitative measurement of VAT using an imaging technique) was not performed in the present study.

In conclusion, this study is the first to demonstrate that the VAT of MUO individuals has a less favourable inflammatory profile than that of MHO individuals as a result of the infiltration of ATMs with increased caspase- 1 activity and IL-1 $\beta$ production. The inflammasome, and particularly the caspase1 , might represent a useful therapeutic target in the treatment and prevention of obesity-induced insulin resistance.

Acknowledgements We thank the study participants and the surgeons of the University Hospital of Liege who assisted with adipose tissue collection. We are grateful to Jenny DeFlines (Division of Diabetes, Nutrition and Metabolic diseases, CHU of Liège, Belgium) for her help in recruiting patients, Raafat Stephan and Sandra Ormenese for flow cytometry analyses (Imaging GIGA-R technological platform, University of Liège, Belgium), Nathalie Renotte (Cellular and Molecular Epigenetic
Laboratory, GIGA, University of Liège, Belgium) and Cédric Lassence (GIGA ST, University of Liège, Belgium) for their technical assistance, Yvette Habraken (GIGA ST, University of Liège, Belgium) for helpful discussion, Xavier Thiry (HEC, University of Liège, Belgium) for his help designing the figures and Pierre J. Lefèbvre (University of Liège, Belgium) for proofreading and excellent suggestions.

Funding This work was funded by grants from FRS-FNRS (grant number 1.A 613.11), GlaxoSmithKline, the Leon Fredericq Foundation and the Rotary Foundation of Liege.

Duality of interest NE, SLP and JP are Research Fellow, Research Associate and Research Director, respectively, of the FNRS. LL is a Research Fellow from the IAP P7/32. Otherwise, the authors declare that there is no duality of interest associated with this manuscript.

Contribution statement All authors contributed to the conception and design of the study, the acquisition, analysis and interpretation of data and the drafting and editing of the manuscript. All of the authors approved the final version of the manuscript.

\section{References}

1. Alberti KG, Eckel RH, Grundy SM et al (2009) Harmonizing the metabolic syndrome: a joint interim statement of the International Diabetes Federation Task Force on Epidemiology and Prevention; National Heart, Lung, and Blood Institute; American Heart Association; World Heart Federation; International Atherosclerosis Society; and International Association for the Study of Obesity. Circulation 120:1640-1645

2. Karelis AD, St-Pierre DH, Conus F, Rabasa-Lhoreth R, Poehlman ET (2004) Metabolic and body composition factors in subgroups of obesity: what do we know? J Clin Endocrinol Metab 89:2569-2575

3. Wildman RP, Muntner P, Reynolds K et al (2008) The obese without cardiometabolic risk factor clustering and the normal weight with cardiometabolic risk factor clustering: prevalence and correlates of 2 phenotypes among the US population (NHANES 1999-2004). Arch Intern Med 168:1617-1624

4. Stefan N, Kantartzis K, Macham J et al (2008) Identification and characterization of metabolically benign obesity in humans. Arch Intern Med 168:1609-1616

5. Bradshaw PT, Monda KL, Stevens J (2013) Metabolic syndrome in healthy obese, overweight, and normal weight individuals: the atherosclerosis risk in communities study. Obesity (Silver Spring) 21:203-209

6. Wajchenberg BL (2000) Subcutaneous and visceral adipose tissue: their relation to the metabolic syndrome. Endocr Rev 21:697-738

7. Tchernof A, Després JP (2013) Pathophysiology of human visceral obesity: an update. Physiol Rev 93:359-404

8. Koster A, Stenholm S, Alley DE et al (2010) Body fat distribution and inflammation among obese older adults with and without metabolic syndrome. Obesity (Silver Spring) 18:2354-2361

9. Fox CS, Massaro JM, Hoffmann U et al (2007) Abdominal visceral and subcutaneous adipose tissue compartments: association with metabolic risk factors in the Framingham Heart Study. Circulation 116:39-48

10. Lundgren M, Svensson M, Lindmark S et al (2007) Fat cell enlargement is an independent marker of insulin resistance and 'hyperleptinaemia'. Diabetologia 50:625-633

11. Weyer C, Foley JE, Bogardus C et al (2000) Enlarged subcutaneous abdominal adipocyte size but not obesity itself, predicts type II diabetes independent of insulin resistance. Diabetologia 43:1498-1506 
12. Gossens GH (2008) The role of adipose tissue dysfunction in the pathogenesis of obesity-related insulin resistance. Physiol Behav 94:206-218

13. Shoelson SE, Lee J (2006) Inflammation and insulin resistance. J Clin Invest 116:1793-1801

14. Hotamisligil GS (2006) Inflammation and metabolic disorders. Nature 444:860-867

15. Donath MT, Shoelson SE (2011) Type 2 diabetes as an inflammatory disease. Nat Rev Immunol 11:98-107

16. Olefsky JM, Glass CK (2010) Macrophages, inflammation, and insulin resistance. Annu Rev Physiol 72:219-246

17. Chawla A, Nguyen KD, Goh YP (2011) Macrophage-mediated inflammation in metabolic disease. Nat Rev Immunol 11:738-749

18. Feve B, Bastard JP (2009) The role of interleukins in insulin resistance and type 2 diabetes mellitus. Nature Rev Endocrinol 5:305-311

19. Osborn O, Olefsky JM (2012) The cellular and signaling networks linking the immune system and metabolism in disease. Nat Med $18: 363-374$

20. Wentworth JM, Naselli G, Brown WA et al (2010) Pro-inflammatory $\mathrm{CD} 11 \mathrm{c}^{+} \mathrm{CD} 206^{+}$adipose tissue macrophages are associated with insulin resistance in human obesity. Diabetes 59:1648-1656

21. Fujisaka S, Usui I, Bukhari A et al (2009) Regulatory mechanisms for adipose tissue M1 and M2 macrophages in diet-induced obese mice. Diabetes 58:2574-2582

22. Patsouris D, Li PP, Thapar D et al (2008) Ablation of CD11 c-positive cells normalizes insulin sensitivity in obese insulin resistant animals. Cell Metab 8:301-309

23. Feuerer M, Herrero L, Cipolletta D et al (2009) Lean, but not obese, fat is enriched for a unique population of regulatory T cells that affect metabolic parameters. Nat Med 15:930-939

24. Nishimura S, Manabe I, Nagasaki M et al (2009) CD8+ effector $\mathrm{T}$ cells contribute to macrophages recruitment and adipose tissue inflammation in obesity. Nat Med 15:914-920

25. Winer S, Chan Y, Paltser G et al (2009) Normalization of obesityassociated insulin resistance through immunotherapy. Nat Med 15:921-929

26. Spranger J, Kroke A, Möhlig M et al (2003) Inflammatory cytokines and the risk to develop type 2 diabetes: results of the prospective population-based European Prospective Investigation into Cancer and Nutrition (EPIC)-Potsdam Study. Diabetes 52:812-817

27. Thorand B, Kolb H, Baumert J et al (2005) Elevated levels of interleukin-18 predict the development of type 2 diabetes. Results from the MONICA/KORA Augsburg study, 1984-2002. Diabetes 54:2932-2938

28. Schroder K, Tschopp J (2010) The inflammasomes. Cell 140:821-832

29. Stienstra R, Tack CJ, Kanneganti TD, Joosten LAB, Netea MG (2012) The inflammasome puts obesity in the danger zone. Cell Metab 15:10-18

30. Vandanmagsar B, Youm YH, Ravussin A et al (2011) The NLRP3 inflammasome instigates obesity-induced inflammation and insulin resistance. Nat Med 17:179-188
31. Lee HM, Kim JJ, Kim HJ et al (2013) Upregulated NLRP3 inflammasome activation in patients with type 2 diabetes. Diabetes 62:194-204

32. Koenen TB, Stienstra R, van Tits LJ et al (2011) The inflammasome and caspase-1 activation: a new mechanism underlying increased inflammatory activity in human visceral adipose tissue. Endocrinology 152:3769-3778

33. Livak KJ, Schmitthen TD (2001) Analysis of relative gene expression data using real-time quantitative PCR and the 2(-Delta Delta $\mathrm{C}(\mathrm{T}))$ method. Methods 25:402-408

34. Arend WP, Palmer G, Gabay C (2008) IL-1, IL-18, and IL-33 families of cytokines. Immunol Rev 223:20-38

35. Schertzer JD, Klip A (2011) Give a NOD to insulin resistance. Am J Physiol Endocrinol Metab 301:E585-E586

36. Kim MS, Choi M-S, Han SN (2011) High fat diet-induced obesity leads to proinflammatory response associated with higher expression of NOD2 protein. Nutr Res Pract 5:219-223

37. Deiuliis J, Shah Z, Shah N et al (2011) Visceral adipose inflammation in obesity is associated with critical alterations in $\mathrm{T}$ regulatory cell numbers. PLoS One 6:e16376

38. Greenberg AS, Obin MS (2006) Obesity and the role of adipose tissue in inflammation and metabolism. Am J Clin Nutr 83:461S$465 \mathrm{~S}$

39. Fried SK, Bunkin DA, Greenberg AS (1998) Omental and subcutaneous adipose tissues of obese subjects release interleukin-6: depot difference and regulation by glucocorticoid. J Clin Endocrinol Metab 83:847-850

40. Membrez M, Ammon-Zufferay C, Philippe D et al (2008) Interleukin-18 protein level is upregulated in adipose tissue of obese mice. Obesity (Silver Spring) 17:393-395

41. Skurk T, Kolb H, Muller-Scholze S et al (2005) The proatherogenic cytokine interleukin-18 is secreted by human adipocytes. Eur J Endocrinol 152:863-868

42. Poulain-Godefroy O, Lecoeur C, Pattou F et al (2008) Inflammation is associated with a decrease of lipogenic factors in omental fat in women. Am J Physiol Regul Integr Comp Physiol 295:R1-R7

43. Lemieux I, Poirier P, Bergeron J et al (2007) Hypertriglyceridemic waist: a useful screening phenotype in preventive cardiology? Can J Cardiol 23(suppl B):23B-31B

44. Shi H, Kokoeva M, Inouye $\mathrm{K}$ et al (2006) TLR4 links innate immunity and fatty acid-induced insulin resistance. J Clin Invest 116:30153025

45. Wen H, Gris D, Lei Y et al (2011) Fatty acid-induced NLRP3-ASC inflammasomes activation interferes with insulin signaling. Nat Immunol 12:408-415

46. Oh DY, Talukdar S, Baz EJ et al (2010) GPR120 is an omega-3 fatty acid receptor mediating potent anti-inflammatory and insulin -sensitizing effects. Cell 142:687-698

47. Chatzigeorgiou A, Karalis KP, Bornstein SR, Chavakis T (2012) Lymphocytes in obesity-related adipose tissues inflammation. Diabetologia 55:2583-2592 